\title{
Developmental Adaptation of Rat Nociceptive Withdrawal Reflexes after Neonatal Tendon Transfer
}

\author{
Hans Holmberg, Jens Schouenborg, Yong-Bei Yu, and Han-Rong Weng \\ Department of Physiology and Neuroscience, University of Lund, S-223 62 Lund, Sweden
}

Nociceptive withdrawal reflexes (NWRs) were studied in adult rats in which the movement patterns produced by single muscles had been altered by neonatal tendon transfer. NWRs evoked by cutaneous noxious mechanical and thermal $\left(\mathrm{CO}_{2}-\right.$ laser) stimulation were recorded using electromyography in a decerebrate spinal preparation. The sensitivity distribution within the receptive fields of the NWRs of the extensor digitorum longus and the peronei muscles exhibited changes corresponding to the altered movement patterns. No detectable change of NWRs was found in normal muscles whose receptive fields overlapped that of the modified muscle. Furthermore, NWRs of muscles that regained an essentially normal function after neonatal tendon transfer did not differ from normal. It is proposed that a developmental experience-dependent mechanism, which takes into account the hindlimb movement pattern caused by contraction of single muscles, underlies the functionally adapted organization of adult NWRs.

Key words: pain; plasticity; development; sensorimotor integration; activity-dependent learning; spinal reflexes
A fundamental function of the adult nervous system is to execute adequate nocifensive motor responses (Sherrington, 1910; Hagbarth, 1952; Willer, 1977; Carew et al., 1983; Schouenborg and Kalliomäki, 1990). Successful accomplishment of this task requires transformation of the stimulus location into appropriate adjustments of muscle activity. To this end, the neuronal connections within nociceptive motor systems must be adapted to the three-dimensional shape of the body and the kinetics of the musculoskeletal apparatus (Schouenborg and Weng, 1994). Clarification of how such specific connections are formed will lead to an increased understanding of the organization and function of the adult nociceptive systems and of sensorimotor reorganization after injury and reconstructive surgery (Sanes et al., 1990; Garraghty and Kaas, 1991; Merzenich and Jenkins, 1993; Schady et al., 1994; Holmberg and Schouenborg, 1996b).

In adult rats, the nociceptive withdrawal reflexes (NWRs) seem to have a "modular" organization, with each reflex pathway essentially controlling either a single or a small group of synergistic muscles (Schouenborg and Kalliomäki, 1990; Schouenborg et al., 1992, 1994a; Schouenborg and Weng, 1994). The location of the cutaneous excitatory receptive field of a reflex pathway, and the distribution of sensitivity within this field, directly reflects the withdrawal movement pattern produced by the effectuating muscle(s) in the normal standing position. For example, maximal reflex responses in a single muscle are evoked from the skin area that is most effectively withdrawn from the stimulation as the muscle contracts. Therefore, the withdrawal movement pattern

Received July 30, 1996; revised Nov. 20, 1996; accepted Dec. 20, 1996.

This work was supported by the Swedish Medical Research Council, Projects No. 10569 and No. 1013, the Medical Faculty of Lund, Swedish Society for Medical Research, Dr. P. Håkanssons Stiftelse, The Royal Physiographical Society in Lund, Elsa and Thorsten Segerfalks Stiftelse, Crafoords Stiftelse, Magn. Bergvalls Stiftelse, Greta and Johan Kocks Stiftelse, and Maggie Stephens Stiftelse.

Correspondence should be addressed to Dr. Jens Schouenborg, Department of Physiology and Neuroscience, University of Lund, Sölvegatan 19, S-223 62 Lund, Sweden.

Dr. Yu's present address: Department of Pharmacology, 13th Biomedical Science Tower, University of Pittsburgh, Pittsburgh, PA, 15260.

Copyright (C) 1997 Society for Neuroscience 0270-6474/97/172071-08\$05.00/0 produced by the effector muscle(s) of a given reflex module is, in a sense, "imprinted" on that module.

NWRs evoked by cutaneous stimulation are functionally unadapted in neonatal rats and often lead to movements directed toward the stimulation (Holmberg and Schouenborg, 1996a). The adult, task-specific NWR organization then gradually emerges over the first three postnatal weeks. Several lines of evidence suggest that this postnatal maturation of reflex patterns reflects a tuning of spinal connectivity. First, the response properties and somatotopic termination pattern of thin $\mathrm{A}$ and $\mathrm{C}$ afferent fibers already appear adult-like at birth in rats (Fitzgerald and Swett, 1983; Smith, 1983; Fitzgerald, 1987a,b,c). Second, at this age, motoneurons synapse with their final target muscles (Kelly, 1983), and electrotonic coupling between heteronymous $\alpha$-motoneurons does not seem to be present (Fulton et al., 1980; Walton and Navarrete, 1991). Third, hindlimb NWRs are near normal in adult rats whose plantar skin innervation has been altered by neonatal transection of the plantar nerves (Holmberg and Schouenborg, 1996b).

We have suggested that the cutaneous sensory feedback ensuing on contraction of single muscles is instrumental in the postnatal tuning of the NWR (Schouenborg and Weng, 1994; Holmberg and Schouenborg, 1996a,b). To evaluate this hypothesis, we have now altered the movement pattern ensuing on contraction of single muscles in newborn rats. The NWR receptive fields of the modified muscles were then mapped when the rats had reached adulthood.

Preliminary findings have been published previously (Schouenborg et al., 1994a).

\section{MATERIALS AND METHODS}

\section{Animals used}

Twenty Wistar rats of both sexes were used. They received food and water ad libitum and were kept in a $12 \mathrm{hr}$ light/dark cycle at a constant environmental temperature of $21^{\circ} \mathrm{C}$ (humidity $65 \%$ ). Supplementary data from normal adult rats were obtained from previous studies in which identical experimental procedures were used $(n=18$ rats; Holmberg and 


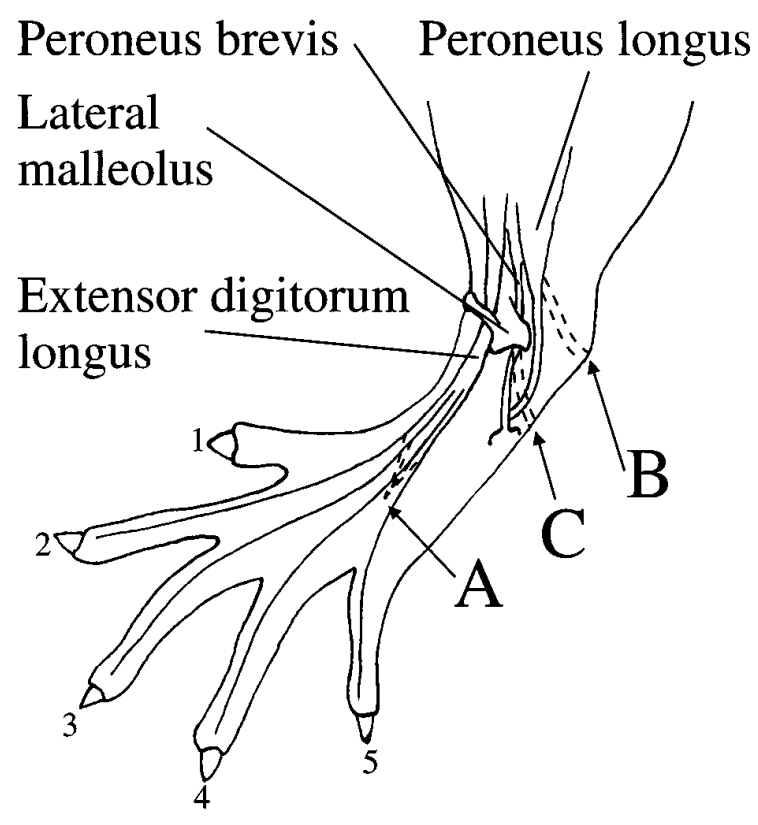

Figure 1. Schematic to show the rearrangement of tendons used. Dorsolateral view of the lower hindlimb. The normal course of tendons is indicated by continuous lines. The tendon of peroneus longus passes on the plantar side of the paw to its insertion point on the first metatarsal bone (not indicated). The course of the tendons after transfer (determined by postmortem examination) is indicated with dashed lines. The different types of tendon transfer are shown in the same schematic for convenience. Arrows indicate new insertion sites for Extensor digitorum longus $(A)$, Peroneus longus and Peroneus brevis, which caused plantar flexion of the ankle after transfer $(B)$, and Peroneus longus and Peroneus brevis, which continued to cause pronation of the hindpaw after transfer $(C)$.

Schouenborg, 1996a,b). Approval for the experiments was obtained from the regional ethical committee in Lund/Malmö.

\section{Neonatal surgery}

The withdrawal reflexes of the muscles peroneus longus and brevis (PER) and extensor digitorum longus (EDL) were chosen for manipulation, because their receptive fields exhibit little variation across normal rats (Schouenborg et al., 1992; Schouenborg and Weng, 1994; Holmberg and Schouenborg, 1996a,b). Also, these muscles have long tendons that can be transferred to new positions at birth.

The rats were removed from the home cage on the day of birth (4-24 $\mathrm{hr}$ after birth) and anesthetized by hypothermia (cooling on ice). Three or four pups from each litter were used. Careful infiltration with $2.0 \mathrm{mg} / \mathrm{ml}$ lignocaine (xylocaine) with $1.2 \mu \mathrm{g} / \mathrm{ml}$ adrenaline was carried out before surgery to reduce nociceptive input and minimize bleeding. Surgery commenced when spontaneous movements had ceased and when no reflexes could be evoked by pinching the skin. The distal tendons of either EDL or PER were exposed through a small skin incision (usually $<4 \mathrm{~mm}$ long). These tendon stumps were attached to the bones with fine resorbable thread (Vicryl 8-0, GS-9; Ethicon, Norderstedt, Germany). Skin incisions were closed with Nobecutan wound spray (Astra Tech, Mölndal, Sweden).

Manipulation of the EDL tendons. To change the principal action of the EDL from digit dorsiflexion to ankle dorsiflexion, the tendons of EDL were cut over the metatarsal phalangeal joint and inserted in the dorsal side of the third and fourth metatarsal bone (Fig. $1 A$ ). In the first two rats in this group, the tendon stumps distal to the new insertion site were not removed. These tendon stumps were found to cross-bridge the metatarsophalangeal and interphalangeal joints in the adult rat, thereby causing considerable stiffening of these joints. To prevent this effect, the tendon stumps distal to the new insertion site were removed in nine rats.

Manipulation of the PER tendons. To change the principal action of PER from pronation of the paw (rotation around the proximodistal axis of the paw in a direction causing the plantar side to face laterally when starting from the standing position) to ankle plantar flexion, the tendons were transferred to the lateral side of the calcaneus bone in three rats
(Fig. $1 B$ ). In two other rats, the tendon of peroneus brevis was transferred to the calcaneous bone (Fig. $1 B$ ), and the tendon of the peroneus longus was transferred to the lateral side of the foot (Fig. 1C). The intention was to change the action of peroneus brevis to ankle plantar flexion, whereas the peroneus longus would continue to cause pronation of the paw. This way, the possibility of unspecific changes of reflex transmission caused by the neonatal surgery itself could be evaluated. In four rats, an attempt was made to change the principal action of PER from pronation to supination by transferring the tendons of the peroneus longus and brevis muscles to the dorsal side of the first metatarsal bone (not indicated).

Recovery after neonatal surgery. The rats were allowed to recover in a temperature-controlled environment and were returned to the home cage after regaining normal body temperature (measured by a noncontact infrared detecting probe; Thermonitor C-1600M, Linear Laboratories, Los Altos, CA). All rats recovered uneventfully and did not exhibit any signs of suffering (such as vocalization, writhing, immobilization, or sustained flexion of the manipulated limb) during recovery from surgery. The growth and behavior of the operated pups were indistinguishable from those of unoperated littermates (monitored daily until the day of the acute experiment), and no signs of infection were observed.

\section{Surgery and preparation in adult rats}

After the rats had reached adulthood (10-16 weeks), they were anesthetized with halothane $(1.0-2.0 \%)$, in a mixture of $65 \%$ nitrous oxide and $35 \%$ oxygen, and were ventilated artificially via a tracheal cannula. The expiratory $\mathrm{CO}_{2}(3.0-4.5 \%)$ was monitored continuously. An infusion of $30-50 \mu \mathrm{l} / \mathrm{min}$ of $5 \%$ glucose in Ringer's acetate, $\mathrm{pH} 7.0$, was administered via the right jugular vein. Mean arterial blood pressure $(75-140 \mathrm{mmHg})$ was monitored continuously in the right brachial artery. Core temperature was maintained between 36.5 and $38.5^{\circ} \mathrm{C}$ using a thermostatically controlled, feedback-regulated heating system. Local infiltration of lignocaine (xylocaine) with adrenaline (concentrations as above) was used to reduce nociceptive input during surgery and to minimize possible postoperative excitability changes (Clarke and Matthews, 1990). A laminectomy of the tenth thoracic vertebrae and a craniotomy were performed, and the rats were decerebrated by transecting the brain stem intercollicularly. The anesthesia was then discontinued, and the exposed spinal cord was transected with a pair of fine scissors. The electroencephalogram, recorded from the right parietal cortex, was dominated by largeamplitude $(0.5-1.5 \mathrm{mV}) \delta$ waves $(1-3 \mathrm{~Hz})$, which demonstrated a comatose state. Immediately after spinalization, a small incision was made in the skin overlying the investigated muscles to facilitate insertion of the needles used for electromyography (EMG) into the muscle bellies (see below). Experiments were terminated on signs of deterioration, i.e., precipitous drops in blood pressure or expiratory $\mathrm{CO}_{2}$ levels. After termination of the experiments, the animals were given a lethal dose of halothane ( $5 \%$ for $>15 \mathrm{~min})$.

\section{EMG recordings}

Reflex responses were recorded with etched, fine steel electrodes (insulated up to $50 \mu \mathrm{m}$ from the tip; diameter at the distal end of insulation, $30-40 \mu \mathrm{m}$; tip diameter, $<3 \mu \mathrm{m}$; length of the electrode, $14 \mathrm{~mm}$; weight, $5 \mathrm{mg}$ ) inserted into the belly of the muscles. Each electrode was soldered to a delicate and flexible copper wire (diameter, $0.08 \mathrm{~mm}$ ). Reference electrodes were placed in the adjacent skin. Recordings were made from the manipulated muscle, and also, in some rats, from a normal ipsilateral hindlimb muscle. To reduce the risk of contamination of the recordings by responses of nearby muscles, care was taken to ensure that the recording electrodes were placed centrally in the muscle bellies. This was confirmed by electrical stimulation through the recording electrodes (Schouenborg et al., 1992). In no case was the threshold current needed to activate nearby muscles $<10 \times$ that required for the muscle under study.

\section{Mapping of cutaneous excitatory receptive fields}

Calibrated noxious pinch and $\mathrm{CO}_{2}$-laser stimulation (Directed Energy, Irvine, CA) (unfocused beam; diameter, $1.1 \mathrm{~mm}$ ) of between 25 and 40 sites on the plantar hindpaw skin was used to map the cutaneous excitatory receptive fields of EDL and PER. Mappings of mechanonociceptive receptive fields were initiated $2 \mathrm{hr}$ after spinalization. The flat surface of a calibrated pinching device $\left(1 \mathrm{~mm}^{2}\right.$ on each side) was applied to a $4 \mathrm{~mm}^{2}$ skin flap, and the pinch force was increased slowly $(\sim 1 \mathrm{~N} / \mathrm{sec})$ and maintained between 2.0 and $2.5 \mathrm{~N}$ for $>1 \mathrm{sec}$ (Schouenborg et al., 1992). Mappings of thermonociceptive receptive fields with $\mathrm{CO}_{2}$-laser stimula- 


\section{Normal EDL}
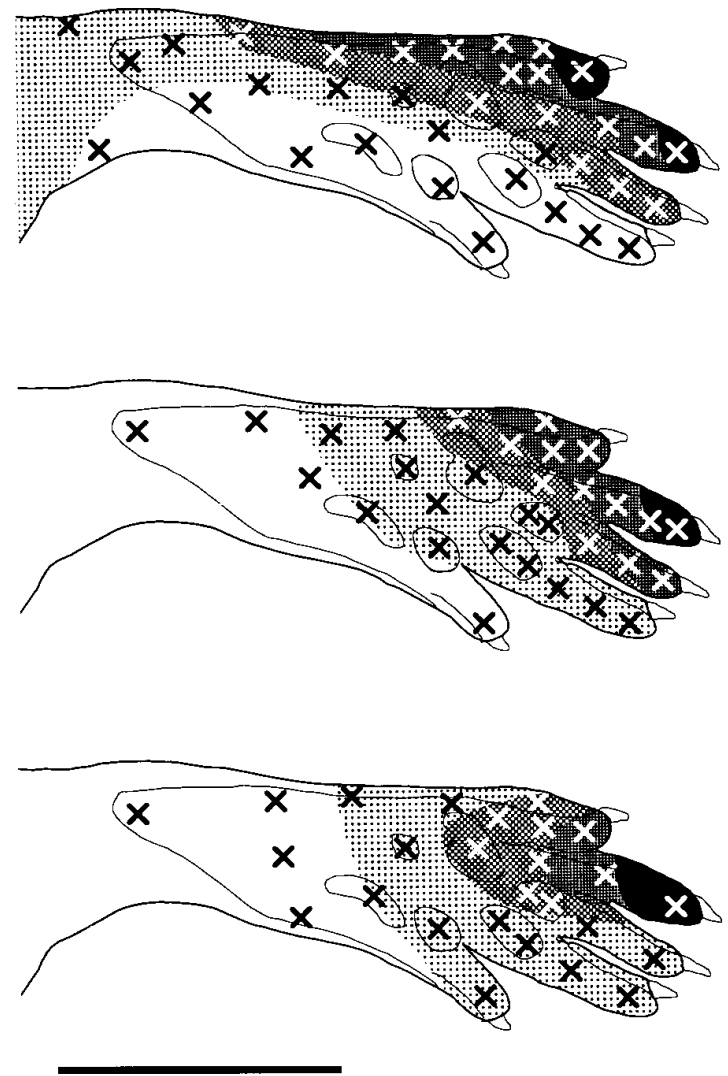

\section{EDL tendons transferred to metatarsal 3 and 4}
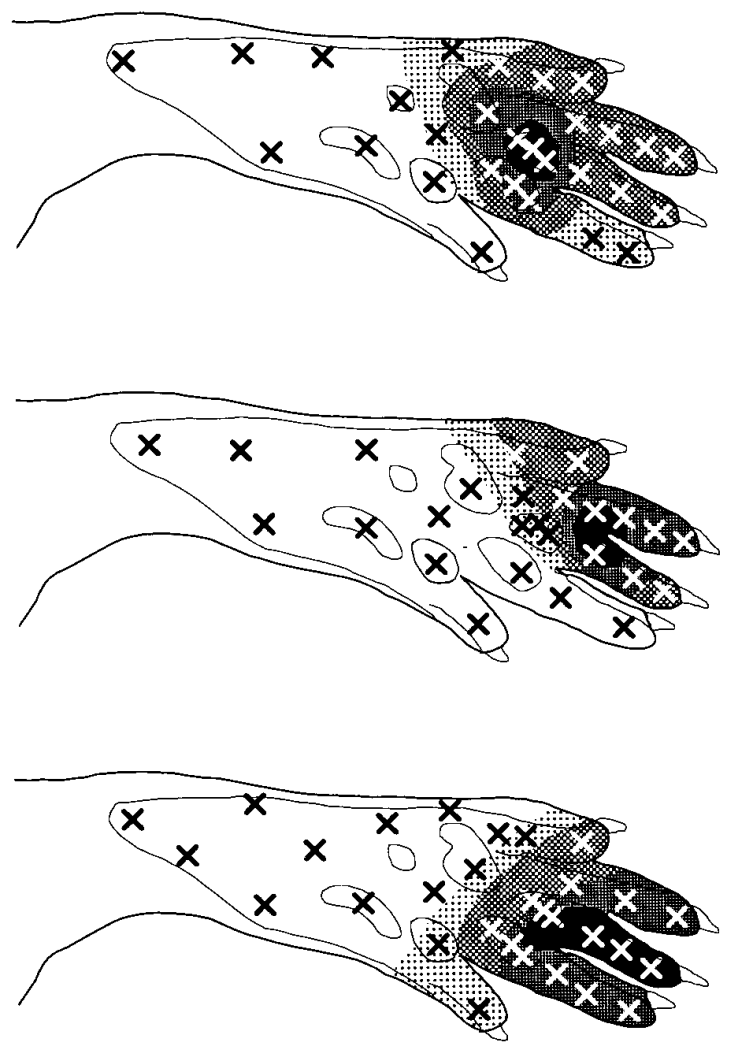

Figure 2. Samples of receptive fields of EDL withdrawal reflexes in three normal rats (left) and in three rats whose EDL tendons were transferred to the third and fourth metatarsal bone at birth (right). Each site was stimulated once with calibrated noxious pinch. The magnitude of the evoked reflex responses is represented by the density of dots. Low, medium, and high dot densities indicate areas of the skin from which the evoked responses were $0-30 \%, 30-60 \%$, and $60-85 \%$ of maximal responses, respectively. Black represents $85-100 \%$ of maximal responses (referred to as receptive field focus). The extent of the respective areas was calculated from the original response amplitudes using a Kriging algorithm (for details, see Materials and Methods). Crosses indicate stimulation sites. Scale bar, $20 \mathrm{~mm}$.

tion (intensity $2 \times$ reflex threshold, $1 \mathrm{~W}, 20-25 \mathrm{msec}$ ) started $4.5 \mathrm{hr}$ after spinalization. Interstimulus intervals were $\sim 1$ min during both types of receptive field mapping.

\section{Analysis}

The magnitude of the reflex responses was defined as the number of clearly distinguishable motor unit spikes (i.e., spikes that could be separated from background noise and electrocardiography deflections) evoked during the first second after the onset of the $\mathrm{CO}_{2}$-laser pulse or during the first second of constant pinch force (spike numbers were counted using the "EGAA program," RC Electronics, Goleta, CA).

To quantitatively describe the receptive field of the withdrawal reflexes of a muscle, the responses in this muscle evoked by stimulation of the plantar side of the foot were normalized and expressed as the percentage of the maximal response in the respective muscle and rat. The normalized values were plotted on the corresponding stimulation sites on a standard diagram of the hindpaw. A receptive field, divided into four areas of differing sensitivity [85-100\% (referred to as receptive field focus), 60 $85 \%, 30-60 \%$, and $0-30 \%$ of maximal response] was then delineated. The areas of differing sensitivity were delineated with the aid of computer-generated isoresponse lines (Kriging algorithm and contour program, software from Golden, Inc., Golden, CO; "Grid" and "Topo" programs; also see Schouenborg et al., 1995).

To quantitatively describe the average receptive field of a muscle in an experimental group, responses evoked by stimulation of the plantar side of the hindpaw were normalized for each muscle in each rat and expressed as the percentage of the maximal response in the respective muscle and rat. For each stimulated site, a mean response value was then calculated and plotted on a standard diagram of the hindpaw. An average receptive field was then delineated and represented as described above.

\section{Statistical analysis}

The two-tailed Mann-Whitney $U$ test was used for statistical evaluation. Differences were considered statistically significant at the level of $p<$ 0.05 . Values are presented as mean \pm SEM.

\section{RESULTS}

\section{Transfer of EDL tendons}

In nine rats, which were subjected to neonatal EDL tendon transfer and removal of the tendon stumps distal to the new insertion, contraction of the modified EDL caused ankle dorsiflexion (confirmed by intramuscular electrical stimulation). Although no active digit movements were present, the digits tended to plantarflex on dorsiflexion of the ankle. On direct visual examination, it was evident that the area maximally withdrawn by this 


\section{Normal rats}

\section{EDL}

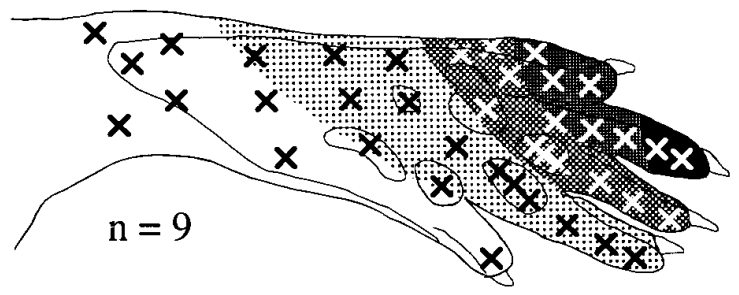

PER

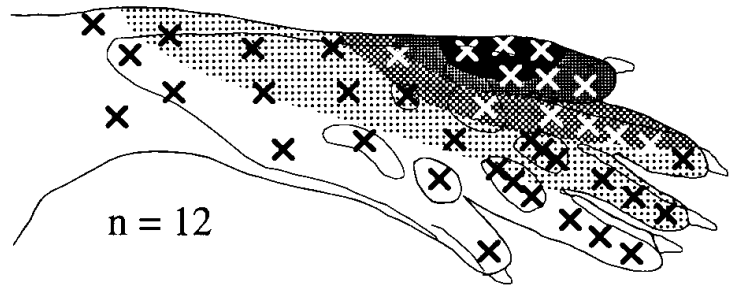

\section{EDL tendons transferred to metatarsal 3 and 4}

EDL

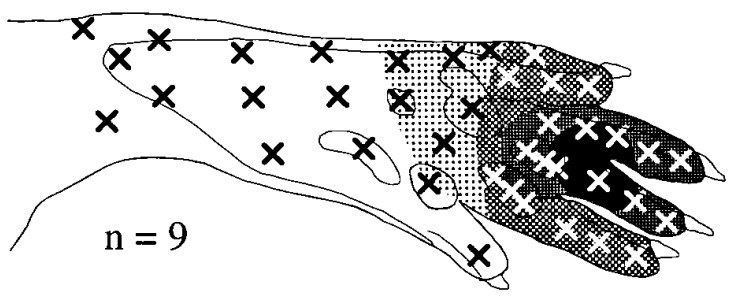

PER

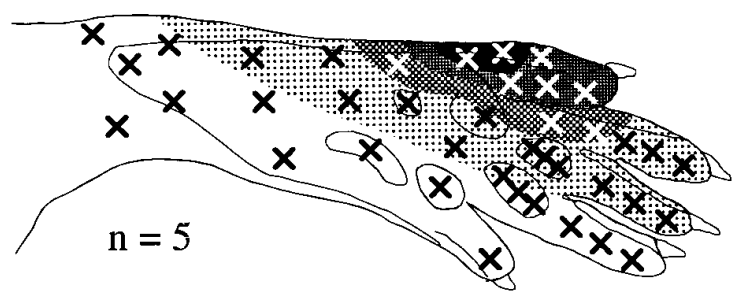

Figure 3. Average receptive fields (for details, see Materials and Methods) of EDL and PER withdrawal reflexes in normal rats (top) and in rats whose EDL tendons were transferred to the third and fourth metatarsal bone at birth (bottom). Receptive field mapping and conventions as in Figure 2.

movement was the plantar skin covering the proximal phalanxes of digits 3 and 4, and the distal part of the metatarsal bones 3 and 4. By comparison, normal EDL tendons cause maximal withdrawal of the plantar skin of the distal part of the digits (Schouenborg and Weng, 1994).

Within the two groups of rats (normal and modified EDL, respectively), only small differences in spatial organization of the receptive fields of EDL were found across animals. Representative receptive fields, as mapped with standardized noxious pinch, are shown in Figure 2. Furthermore, the variability of response magnitude was typically low for each stimulation site. The range of SEM and average SEM for normalized response amplitudes for all stimulation sites within the receptive fields of normal EDL were $0.2-17.5$ and 6.6, respectively. Corresponding values for modified EDL were 0.1-13.6 and 7.2, respectively.

Average receptive fields of normal ( $n=9$ rats) and modified EDL ( $n=9$ rats), both obtained using mechanical stimulation, are shown in Figure 3. Compared with normal EDL, a marked distoproximal shift was observed in the distribution of sensitivity within the receptive field of the modified EDL. In the modified EDL, maximal responses were evoked from the proximal parts of the digits and the distal part of the plantar skin overlying metatarsal bones 3 and 4 . There was a significant increase $(p<0.01)$ of the magnitude of reflexes evoked from this area in modified EDL (123 \pm 20 spikes/sec), as compared with normal EDL (58 \pm 11 spikes/sec). The alteration of receptive field organization of the modified EDL thus corresponded to the tendon transferinduced shift in withdrawal efficacy to more proximal parts of the skin. The magnitude of responses evoked from the distal part of the digits, i.e., the area of maximal sensitivity for reflexes of EDL in normal rats, was unchanged in transferred EDL as compared with normal EDL. The change in the distribution of sensitivity for transferred EDL was confirmed using $\mathrm{CO}_{2}$-laser stimulation in three rats (data not shown).

Reflexes of PER (which had not been modified in these rats) were recorded simultaneously in five of the nine EDL-transferred rats for control purposes. Receptive field organization and response amplitudes of reflexes of PER were indistinguishable from normal, indicating a selective effect on the modified muscle group.

Recordings were also made from two different portions of the modified EDL ( $n=3$ rats), corresponding to the subunits normally acting on digits $2-3$ and $4-5$, respectively (as judged from the position of the muscle bellies). In these two modified EDL subunits, which produced identical hindpaw movements on intramuscular electrical stimulation, maximal responses were evoked from the same area of the skin (Fig. 4). In normal rats, maximal responses in different EDL subunits are evoked from different digits, corresponding to the different withdrawal action of the respective subunits (Schouenborg et al., 1992); however, although the different modified EDL subunits produced the same movement, the receptive field border of the subunits that normally act 


\section{EDL tendons transferred to metatarsal 3 and 4}
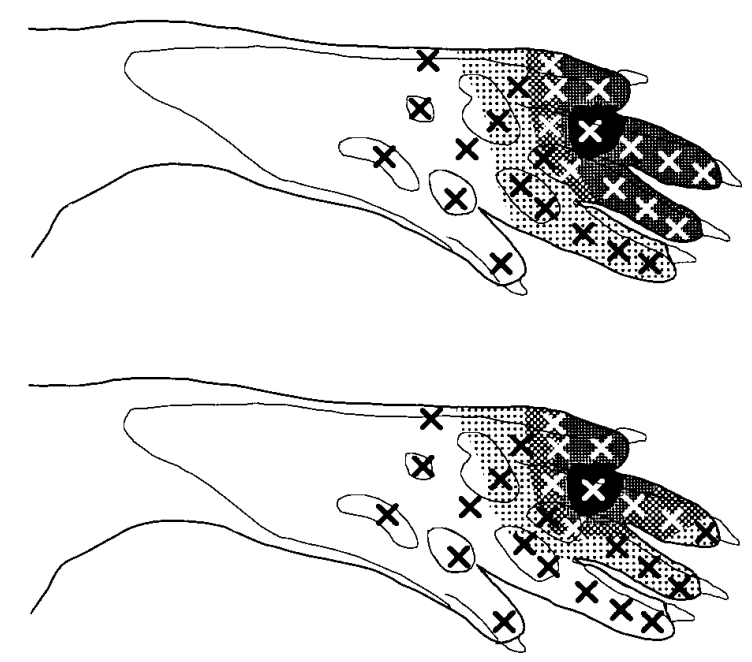

Figure 4. Receptive fields of two simultaneously recorded subunits of EDL, the distal tendons of which were attached to the third and fourth metatarsal bone. Recordings were made from the subunits of EDL, which normally act on digits 2-3 (top) and 4-5 (bottom), respectively, as judged from the position of the EMG electrodes. Data obtained from one rat are shown. Receptive field mapping and conventions as in Figure 2.

on digits $2-3$ was more medially located than that of the subunits that normally act on digits 4-5 (Fig. 4).

\section{Transfer of PER tendons}

In three rats, the principal action of both peroneus longus and peroneus brevis was changed from pronation of the paw to ankle plantar flexion, although a weak pronation was observed on intramuscular electrical stimulation when the rat was in a standing position. The area maximally withdrawn thus changed from the plantar side of digit 5 to the heel. The magnitude of mechanoreceptive reflex responses evoked in the transferred PER was reduced to $\sim 30 \%$ of control $(p<0.05$ ), but the receptive field focus was not shifted to the heel. Only minor differences in spatial organization of the receptive fields of PER with similar withdrawal action were found across animals. Furthermore, as for EDL, the variability of response magnitude was typically low for each stimulation site. The range of SEM and the average SEM for normalized response amplitudes for all stimulation sites within the receptive fields of normal PER were 0.1-9.6 and 4.1, respectively. Corresponding values for modified PER were 1.3-24 and 9.8 , respectively. The receptive field organization and reflexresponse magnitude of reflexes of EDL (simultaneously recorded), which had not been transferred in this group of rats, were not different from normal.

In two other rats, the movement ensuing on contraction of the peroneus brevis was changed to ankle plantar flexion, whereas the peroneus longus continued to cause pronation of the foot. Simultaneous recordings from the two muscles revealed that the reflex response magnitude was clearly lowered in the plantar flexing muscle, whereas it was not appreciably different from normal in the muscle producing pronation (Fig. $5 A$ ). In normal rats, there is no systematic difference in reflex response magnitude between these two muscles (Schouenborg et al., 1992). $\mathrm{CO}_{2}$-laser stimulation was used to characterize stimulus-response relationships in these animals. The $\mathrm{CO}_{2}$-laser-evoked responses were reduced dramatically in the modified compared with the nonmodified muscle (Fig. 5B).

\section{Reflexes of manipulated muscles that regained a near-normal function}

In two rats, the transfer of the EDL tendons to the metatarsal bone did not result in a shift of the area maximally withdrawn (Fig. 6). Here, the distal stump of the EDL tendons cross-bridged, and thereby stiffened, the metatarsal-phalangeal and interphalangeal joints. As in normal rats, maximal EDL reflexes were evoked from the distal part of the digits in these rats (Fig. 6).

In four rats, attempts were made to shift the tendons of PER to the first metatarsal bone; however, the tendons of these PER reattached to the lateral and dorsal side of the foot, thus restoring an apparently normal movement pattern on muscle contraction. The reflexes of these muscles did not differ appreciably from normal with regard to magnitude or spatial organization of the receptive field. These findings clearly indicate that the surgical procedure in the neonatal rat did not produce, in itself, longlasting alterations of reflex organization.

\section{DISCUSSION}

In the present study, it was demonstrated that the spinal nociceptive reflex pathways adapt to neonatally induced alterations of movement patterns. Thus, as has been described for many sensory systems (Knudsen, 1985; Singer, 1990; Simon et al., 1992; Schlaggar, 1993; Benedetti and Ferro, 1995), this spinal nociceptive motor system seems to attain its adult organization at least partially through experience-dependent mechanisms. The eventual formation of an "imprint" of the movement pattern on the reflex pathway may indicate a special form of somatosensory "imprinting" mechanisms during development (see below).

The central representations of the body surface may become reorganized after various forms of manipulations, e.g., nerve lesions (Killackey et al., 1994) and limb amputations (Killackey and Dawson, 1989). Although such effects are most pronounced after manipulations performed during early development, central representations can also be modified in the adult (Merzenich et al., 1983; Kaas, 1995; Nudo et al., 1996). It should be noted, however, that the selective change of sensorimotor transformation in the manipulated reflex pathways found in the present study cannot be explained readily by a central change of body representation. Rather, these findings may reflect specific reorganization within the manipulated reflex pathways.

\section{Modular organization of the withdrawal reflexes}

The selective change in the distribution of sensitivity within the receptive field of the manipulated EDL or PER reinforces the notion that the spinal NWRs have a "modular" organization, with each module controlling a single, or a few synergistic, muscle(s) (Schouenborg and Kalliomäki, 1990; Kalliomäki et al., 1992; Schouenborg et al., 1994b). In fact, even the reflex pathways to peroneus brevis and peroneus longus muscles were affected selectively by tendon transfer, suggesting that at least partially separate reflex pathways exist to different, but synergistic, muscles. Weak, and therefore functionally less important, connections between NWR pathways to different muscles cannot be ruled out, however. 


\section{A PL pronation restored}

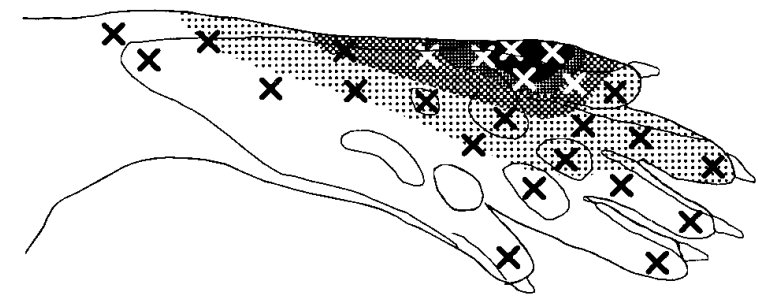

\section{PB ankle plantar flexion}

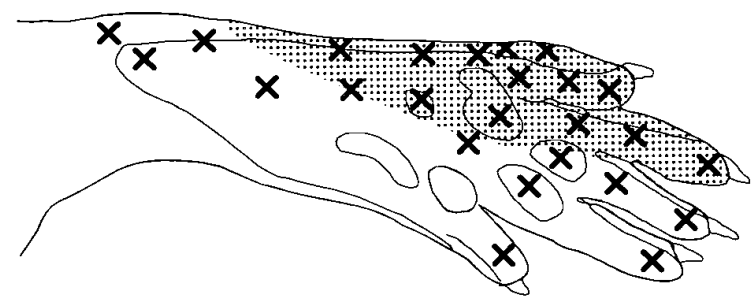

B

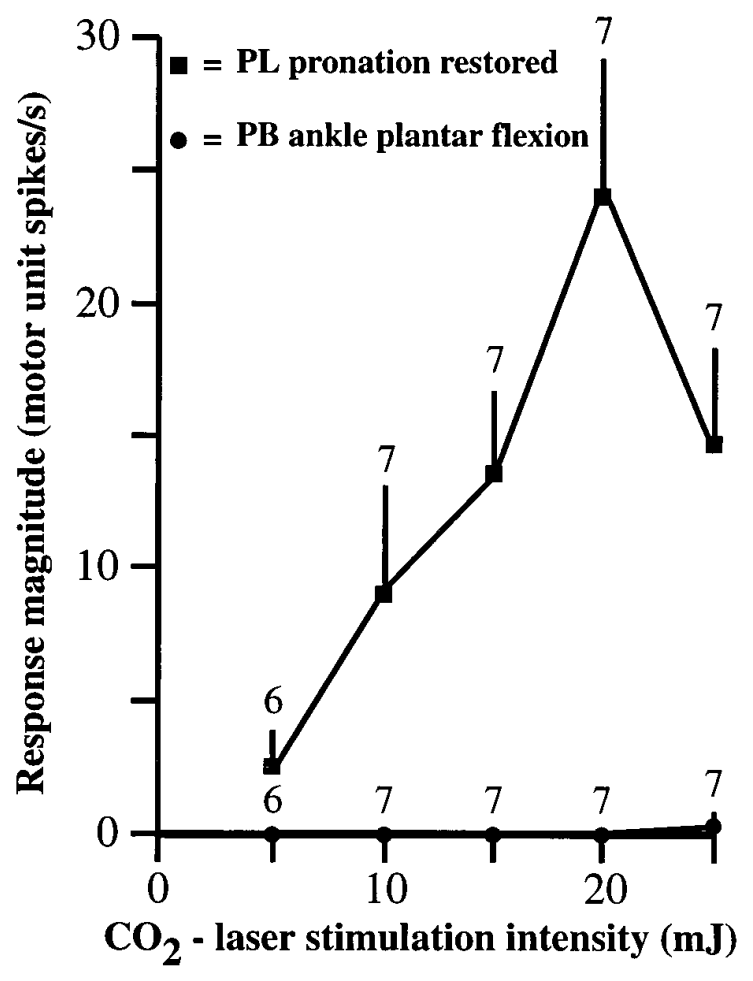

Figure 5. A, Samples of simultaneously mapped receptive fields of a peroneus longus $(P L)$ muscle causing a normal pronation (top), and a modified peroneus brevis $(P B)$ muscle causing ankle plantar flexion and very weak pronation (bottom). Data obtained from one rat are shown. The respective areas of dot densities (see Fig. 2) indicate the reflex response magnitude as a percentage of the maximal reflex response in the peroneus longus muscle. $B$, Graphs showing the stimulus-response relationship of a modified peroneus brevis muscle (causing ankle plantar flexion and very weak pronation) and a simultaneously recorded peroneus longus muscle (causing normal pronation). $\mathrm{CO}_{2}$-laser stimulation was used. Data from one rat are shown. Stimulation sites are indicated with crosses.

\section{Adaptation of withdrawal reflex pathways to altered input-output relationships}

Neonatal tenotomy has been shown to cause a delay in the developmental regression of the muscle fiber multi-innervation present in early development, but a normal pattern of muscle innervation is eventually established after healing of the tendon (Redfern, 1970; Benoit and Changeux, 1975). In the present study, no significant effects on withdrawal reflexes were observed when a near-normal movement pattern was regained after tendon transfer. Effects unrelated to the change of movement pattern, such as the temporary changes in muscle innervation described above, therefore can presumably be ruled out as an explanation for the adaptive changes seen in the present study. Furthermore, reflexes of EDL and PER have overlapping receptive fields and therefore may receive cutaneous input from partially common afferent fibers. Hence, the selective alteration of the distribution of sensitivity within the receptive field of the successfully manipulated EDL indicates that the changes are not attributable to effects on cutaneous hindpaw innervation or to general changes of the somatotopical representation of the body surface.

We recently found that normal receptive fields of the withdrawal reflexes develop despite a profound neonatally induced alteration of the plantar innervation (Holmberg and Schouenborg, 1996b). Thus, during ontogeny, withdrawal reflexes can adapt to early alterations of both movement patterns and peripheral innervation. The present results, however, indicate that limitations exist to this capacity for adaptation. For example, PER, whose tendons were translocated to the calcaneus bone, did not exhibit a novel focus on the heel, i.e., on the skin area maximally withdrawn by the manipulated PER. Furthermore, the location of the receptive field borders of the manipulated PER and EDL muscles appeared unchanged. As in normal rats, the receptive fields of the manipulated EDL subunits that would normally act on digits 2-3 had a more medially located receptive field border than the manipulated EDL subunits that would normally act on digits 4-5 (Fig. 4), despite the fact that both manipulated subunits produced the same movement. These findings may suggest that each individual reflex pathway receives cutaneous input from a given skin area, and that the adult distribution of input strength from this area is the result of a postnatal tuning process. The location and extent of this area could be determined by the spatial relation between the reflex interneurons (Schouenborg et al., 1995) and the location of the primary afferent terminations in the spinal cord dorsal horn (Molander and Grant, 1986).

\section{Mechanisms underlying the postnatal tuning of withdrawal reflex pathways}

A change of movement pattern would produce a corresponding change in the sensory feedback that ensues on muscle contraction, because the feedback from most cutaneous mechanoreceptors reflects the change in load on the skin surface (Fleischer et al., 1983; Willis and Coggeshall, 1991; Leem et al., 1993). The change of receptive fields corresponded to the induced changes of the movement patterns of the manipulated muscles. This supports our 


\section{Normal EDL}

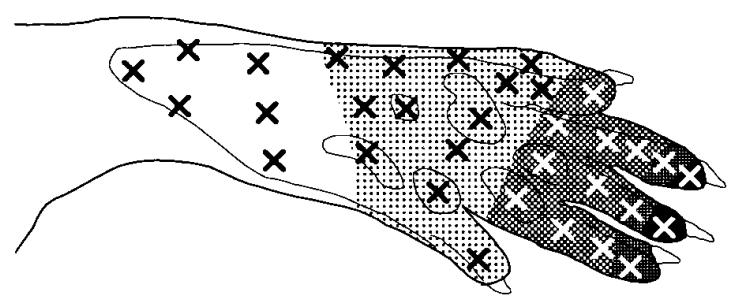

\section{EDL movement partially restored}

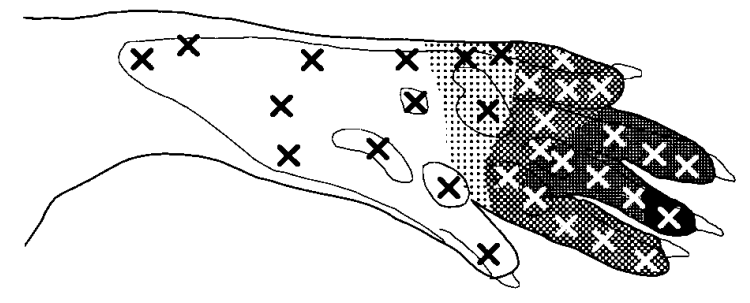

Figure 6. Samples of EDL receptive fields in a normal rat (top) and in a rat in which a normal movement pattern was restored after neonatal surgery (bottom). In the latter EDL, the distal tendon stump crossbridged, and therefore stiffened, the metatarsal-phalangeal and interphalangeal joints. As in normal rats, the distal parts of the digits were therefore maximally withdrawn on EDL contraction. Data obtained in single rats are shown. Receptive field mapping and conventions are as in Figure 2.

hypothesis that the cutaneous sensory feedback is instrumental in strengthening appropriate connections and depressing inappropriate ones (Schouenborg and Weng, 1994; Holmberg and Schouenborg, 1996a,b). This notion differs from, but is not incompatible with, the previous suggestion that the emergence of an adult reflex organization results from a relative strengthening of inhibitory connections over excitatory ones (Ekholm, 1967; Fitzgerald and Koltzenburg, 1986; Fitzgerald, 1991; Guy and Abbott, 1992). In view of the selective alteration of the cutaneous nociceptive input to the manipulated muscles, different reflex modules seem to be tuned independently of one another. The feedback ensuing on contraction of the effector muscle of a given module must therefore be discriminated from the feedback ensuing on contraction of other muscles. This could be accomplished if "spontaneous" excitability fluctuations in reflex interneurons of single reflex modules triggered "test" contractions of their effector muscles. The ensuing feedback then would be temporally correlated with the interneuronal activity within the reflex circuit. It is conceivable that this temporal correlation leads to a selective strengthening of the efficacy of appropriate connections and a reduction in the efficacy of inappropriate ones. Indeed, nonreflexogenic spontaneous limb movements (Hamburger, 1970) are a common feature of normal development and appear around embryonic day 15 in the rat, i.e., at approximately the time at which reflex activity can first be evoked (Angulo y Gonzalez, 1932; Narayanan et al., 1971).

The developmental "sensorimotor autolearning" mechanisms that are proposed to tune the withdrawal reflex circuitry differ from those assumed to underlie the formation of topographically organized sensory maps. In the latter case, numerous studies indicate a crucial role of Hebbian mechanisms, perhaps involving NMDA receptor-mediated detection of temporally correlated synaptic activity (Singer, 1990; Simon et al., 1992; Schlaggar et al., 1993; Scheetz and Constantine-Paton, 1994). Hebb (1949) postulated that the connections between a pre- and postsynaptic neuron may in some cases be strengthened if the probability is high that impulses in the presynaptic neuron are followed by postsynaptic action potentials. Temporally correlated inputs to a common postsynaptic neuron would thereby be consolidated. This then would underlie the developmental transition from initially randomized afferent inputs to topographically organized projections. According to the present hypothesis, the tuning of the spatial organization of the withdrawal reflexes, in contrast, would be initiated by activity in the (postsynaptic) reflex interneurons. This activity would lead, in turn, to muscle twitches and thereby to a temporally correlated sensory feedback. Also, the input efficacy would be increased for the afferents whose activity is reduced and decreased for the afferents whose activity is increased, as a consequence of the movement of the skin surface that follows the "test" muscle contractions. It should be noted that inhibitory mechanisms may play a part in the suggested synaptic plasticity. For example, a decrease in input strength can be attributable to an increase of pre- and/or postsynaptic inhibitory input or a decrease of excitatory input or both.

\section{General aspects}

Apart from the NWRs, movement-related receptive fields have also been described for neurons in the primary motor cortex (Asanuma et al., 1968; Rosén and Asanuma, 1972) and for nociceptive climbing fibers projecting to the anterior cerebellar lobe (Ekerot et al., 1991). It is possible, therefore, that mechanisms similar to those proposed to tune the withdrawal reflexes are involved in the developmental tuning of other motor systems. If so, "spontaneous" movements during prenatal and early postnatal life (Edwards and Edwards, 1970; Pillai and James, 1990) may constitute the observable effects of developmental "test pulses" emitted from different central motor systems.

\section{REFERENCES}

Angulo y Gonzalez AW (1932) The prenatal development of behavior in the albino rat. J Comp Neurol 55:395-442.

Asanuma H, Stoney Jr SD, Abzug C (1968) Relationship between afferent input and motor outflow in cat motorsensory cortex. J Neurophysiol 31:670-681.

Benedetti F, Ferro I (1995) The effects of early postnatal modification of body shape on the somatosensory-visual organization in mouse superior colliculus. Eur J Neurosci 7:412-418.

Benoit P, Changeux J-P (1975) Consequences of tenotomy on the evolution of multiinnervation in developing rat soleus muscle. Brain Res 99:354-358.

Carew TJ, Hawkins RD, Kandel ER (1983) Differential classical conditioning of a defensive withdrawal reflex in Aplysia Californica. Science 219:397-400.

Clarke RW, Matthews B (1990) The thresholds of the jaw-opening reflex and trigeminal brainstem neurons to tooth-pulp stimulation in acutely and chronically prepared cats. Neuroscience 36:105-114.

Edwards DD, Edwards JS (1970) Fetal movement: development and time course. Science 169:95-97.

Ekerot CF, Garwicz M, Schouenborg J (1991) Topography and nociceptive receptive fields of climbing fibers projecting to the cerebellar anterior lobe in the cat. J Physiol (Lond) 441:257-274.

Ekholm J (1967) Postnatal changes in cutaneous reflexes and in the discharge pattern of cutaneous and articular sense organs. Acta Physiol Scand (Suppl) 297:1-130.

Fitzgerald M (1987a) Prenatal growth of fine-diameter primary afferents 
into the rat spinal cord: a transganglionic tracer study. J Comp Neurol 261:98-104.

Fitzgerald M (1987b) Spontaneous and evoked activity of fetal primary afferents in vivo. Nature 326:603-605.

Fitzgerald M (1987c) Cutaneous primary afferent properties in the hind limb of the neonatal rat. J Physiol (Lond) 383:79-92.

Fitzgerald M (1991) The developmental neurobiology of pain. In: Proceedings of the VI Congress on Pain (Bond MR, Charlton JE, Woolf CJ, eds), pp 253-261. Amsterdam: Elsevier Science.

Fitzgerald M, Koltzenburg M (1986) The functional development of descending inhibitory pathways in the dorsolateral funiculus of the newborn rat spinal cord. Dev Brain Res 24:261-270.

Fitzgerald M, Swett J (1983) The termination pattern of sciatic nerve afferents in the substantia gelatinosa of neonatal rats. Neurosci Lett 43:149-154.

Fleischer E, Handwerker HO, Joukhadar S (1983) Unmyelinated nociceptive units in two skin areas of the rat. Brain Res 267:81-92.

Fulton BP, Miledi R, Takahashi T (1980) Electrical synapses between motoneurons in the spinal cord of the newborn rat. Proc R Soc London [Biol] 208:115-120.

Garraghty PE, Kaas JH (1991) Large-scale functional reorganization in adult monkey cortex after peripheral nerve injury. Proc Natl Acad Sci USA 88:6976-6980.

Guy ER, Abbott FV (1992) The behavioral response to formalin in preweanling rats. Pain 51:81-90.

Hagbarth KE (1952) Excitatory and inhibitory skin areas for flexor and extensor motoneurones. Acta Physiol Scand (Suppl) 94:1-58.

Hamburger V (1970) Embryonic motility in vertebrates. In: The neurosciences: second study program (Schmitt FO, ed), pp 141-151. New York: Rockefeller UP.

Hebb DO (1949) The organization of behavior: a neurophysiological theory. New York: Wiley.

Holmberg H, Schouenborg J (1996a) Postnatal development of the nociceptive withdrawal reflexes in the rat: a behavioural and electromyographic study. J Physiol (Lond) 493:239-252.

Holmberg H, Schouenborg J (1996b) Developmental adaptation of withdrawal reflex function following early alteration of peripheral innervation in the rat. J Physiol (Lond) 495:399-409.

Kaas JH (1995) The reorganization of sensory and motor maps in adult mammals. In: The cognitive neurosciences (Gazzanaiga MS, ed), pp 51-71. Boston: MIT.

Kalliomäki J, Schouenborg J, Dickenson AH (1992) Differential effects of a distant noxious stimulus on hindlimb nociceptive withdrawal reflexes in the rat. Eur J Neurosci 4:648-652.

Kelly AM (1983) Emergence of specializations in skeletal muscle. In: Handbook of physiology, Section 10 (Peachey LD, Adrian RH, Geiger SR, eds), pp 507-537. Baltimore: Williams \& Wilkins.

Killackey HP, Dawson DR (1989) Expansion of the central hind paw representation following fetal forelimb removal in the rat. Eur J Neurosci 1:210-221.

Killackey HP, Chiaia NL, Bennet-Clarke CA, Eck M, Rhoades RW (1994) Peripheral influences on the size and organization of somatotopic representations in the fetal rat cortex. J Neurosci 14:1496-1506.

Knudsen EI (1985) Experience alters the spatial tuning of auditory units in the optic tectum during a sensitive period in the barn owl. J Neurosci 5:3094-3109.

Leem JW, Willis WD, Chung JM (1993) Cutaneous sensory receptors in the rat foot. J Neurophysiol 69:1684-1699.

Merzenich MM, Jenkins WM (1993) Reorganization of cortical representations of the hand following alterations of skin inputs induced by nerve injury, skin island transfers, and experience. $J$ Hand Ther 6:89-104.

Merzenich MM, Kaas JH, Wall J, Nelson RJ, Sur M, Felleman D (1983) Topographic reorganization of somatosensory cortical areas $3 \mathrm{~B}$ and 1 in adult monkeys following restricted deafferentation. Neuroscience 8:33-55.

Molander C, Grant G (1986) Laminar distribution and somatotopic organization of primary afferent fibers from hindlimb nerves in the dorsal horn: a study by transganglionic transport of horseradish peroxidase in the rat. Neuroscience 19:297-312.

Narayanan CH, Fox MW, Hamburger V (1971) Prenatal development of spontaneous and evoked activity in the rat. Behavior 40:100-134.

Nudo RJ, Milliken GW, Jenkins WM, Merzenich MM (1996) Usedependent alterations of movement representations in primary motor cortex of adult squirrel monkeys. J Neurosci 16:785-807.

Pillai M, James D (1990) Are the behavioural states of the newborn comparable to those of the fetus? Early Hum Dev 22:39-49.

Redfern PA (1970) Neuromuscular transmission in new-born rats. J Physiol (Lond) 209:701-709.

Rosén I, Asanuma H (1972) Peripheral afferent inputs to the forelimb area of the monkey motor cortex: input-output relations. Brain Res 14:257-273.

Sanes JN, Suner S, Donoghue JP (1990) Dynamic organization of primary motor cortex output to target muscles in adult rats. I. Long-term patterns of reorganization following motor or mixed peripheral nerve lesions. Exp Brain Res 79:479-491.

Schady W, Braune S, Watson S, Torebjörk HE, Schmidt R (1994) Responsiveness of the somatosensory system after nerve injury and amputation in the human hand. Ann Neurol 36:68-75.

Scheetz AJ, Constantine-Paton M (1994) Modulation of NMDA receptor function: implications for vertebrate neural development. FASEB J 8:745-752.

Schlaggar BL, Fox K, O’Leary DDM (1993) Postsynaptic control of plasticity in developing somatosensory cortex. Nature 364:623-626.

Schouenborg J, Kalliomäki J (1990) Functional organization of the nociceptive withdrawal reflexes. I. Activation of hindlimb muscles in the rat. Exp Brain Res 83:67-78.

Schouenborg J, Weng H-R (1994) Sensorimotor transformation in a spinal motor system. Exp Brain Res 100:170-174.

Schouenborg J, Holmberg H, Weng H-R (1992) Functional organization of the nociceptive withdrawal reflexes. II. Changes of excitability and receptive fields after spinalization in the rat. Exp Brain Res 90:469-478.

Schouenborg J, Holmberg H, Yu Y-B, Weng H-R (1994a) The organization of spinal nociceptive withdrawal reflexes depends on ontogenetic learning in the rat. Soc Neurosci Abstr 20:232.2.

Schouenborg J, Weng H-R, Holmberg H (1994b) Modular organization of spinal nociceptive reflexes: review. News Physiol Sci 9:261-265.

Schouenborg J, Weng H-R, Kalliomäki J, Holmberg H (1995) A survey of spinal dorsal horn neurones encoding the spatial organization of withdrawal reflexes in the rat. Exp Brain Res 106:19-27.

Sherrington CS (1910) Flexion-reflex of the limb, crossed extension reflex and reflex stepping and standing. J Physiol (Lond) 40:28-121.

Simon D, Prusky GT, O'Leary DDM, Constantine-Paton M (1992) $N$-methyl-D-aspartate receptor antagonists disrupt the formation of a mammalian neural map. Proc Natl Acad Sci USA 89:10593-10597.

Singer W (1990) Ontogenetic self-organization and learning. In: Brain organization and memory (McGaugh JL, Weinberger NM, Lynch G, eds), pp 211-233. New York: Oxford Science.

Smith CL (1983) The development and postnatal organization of primary afferent projections to the rat thoracic spinal cord. J Comp Neurol 220:29-43.

Walton KD, Navarrette R (1991) Postnatal changes in motoneurone electrotonic coupling studied in the in vitro rat lumbar spinal cord. J Physiol (Lond) 433:283-305.

Willer JC (1977) Comparative study of perceived pain and nociceptive flexion reflex in man. Pain 3:69-80.

Willis WD, Coggeshall RE (1991) Sensory mechanisms of the spinal cord. New York: Plenum. 